\title{
Esgotamento Psicológico no Trabalho: uma análise sob a ótica da Síndrome de Burnout em Professores do Ensino Fundamental
}

\author{
Quenia Lanusse da Silva ${ }^{1}$; Thiago Felix de Almeida Menezes ${ }^{2}$; Fernanda Roda de Souza Araújo Cassundé ${ }^{3}$
}

\begin{abstract}
Resumo: Este artigo teve como finalidade avaliar a ocorrência da síndrome de Burnout nos Professores do Ensino Fundamental, do Colégio da Polícia Militar (CPM) no Vale do São Francisco. Foi realizada uma pesquisa quantitativa e empregado o questionário MBI-ED-Maslash Burnout Inventory - Educators Survey (MASLACH; JACKON; LEITER, 1996), versão para professores, com adaptação para uso no Brasil. A pesquisa foi realizada no CPM Alfredo Vianna, localizado em Juazeiro-Bahia. Foi identificado que todos os professores apresentaram indícios de Burnout, devido principalmente à alta carga horária de trabalho e o estresse diário em sala de aula. Este resultado é preocupante, visto que os professores têm no máximo 10 anos de serviço e ainda terão muitos anos de trabalho. É imprescindível a necessidade de transformação na rotina desses profissionais, como diminuição na carga horária e incentivo para capacitações para diminuição do nível de tensão e estresse. Ao mesmo tempo é importante que cada professor procure profissionais capacitados para auxiliarem a prevenir o agravamento dos sintomas, aprimorando a qualidade no seu desempenho profissional e a sua qualidade de vida.
\end{abstract}

Palavras-chave: Burnout. Professor. Qualidade de vida.

\section{Depletion Psychology at Work: an analysis from the perspective of Burnout Syndrome in Elementary School Teachers}

\begin{abstract}
This article's main purpose is to evaluate the occurrence of Burnout syndrome in Primary Education Teachers, the College of Military Police (CPM) in the São Francisco Valley. a quantitative survey and employee questionnaire MBI-ED-Maslash Burnout Inventory was held - Educators Survey (Maslach, JACKON; Leiter, 1996), version for teachers, with adaptation for use in Brazil. The survey was conducted in CPM Alfredo Vianna, located in Juazeiro, Bahia. It was identified that all teachers showed evidence of Burnout, mainly due to high workload and daily stress in the classroom. Since teachers have more service time they are the ones that are closer to developing Burnout syndrome. This result is alarming, as the teachers have no more than 10 years of service and still have many years of work. It is imperative the need for change in the routine of these professionals, as decrease in workload and encouragement for training to decrease the level of tension and stress. At the same time it is important that every teacher look for qualified professionals to assist to prevent the worsening of symptoms, improving quality in their professional performance and their quality of life.
\end{abstract}

Keywords: Burnout. Teacher. Quality of life.

\footnotetext{
Pós-Graduanda em Gestão Pública pela Universidade Federal do Vale do São Francisco;

${ }^{2}$ Pós-Graduando em Gestão Pública pela Universidade Federal do Vale do São Francisco;

3Doutora em Administração. Professora do Colegiado de Administração da Universidade Federal do Vale do São Francisco;

Contato autor correspondente - E-mail: fernanda.roda@univasf.edu.br
} 


\section{Introdução}

A docência é uma das mais antigas e nobres profissões, é dela que partem as demais. A atividade de docência é essencial para estruturação de uma sociedade e formação de seus indivíduos (LOPES, PONTES, 2009). Segundo Rubega, Pacheco (2000) e Santos (2013) esta profissão é responsável por "formar mão de obra e força de trabalho para a Sociedade Capitalista".

As cobranças impostas ao professor são muitas, desde a transmissão do conteúdo, o gerenciamento dos conflitos em sala de aula e a construção junto aos alunos de uma visão crítica sobre as questões do dia a dia. Observa-se até uma inversão dos papéis, quando algumas competências da educação que deveriam ser dos pais, ficam a cargo do professor.

Se por um lado, todos esperam uma mudança através da educação, colocando o professor como principal responsável por essa transformação, por outro lado, este profissional não tem o reconhecimento merecido, por parte do governo, alunos, pais de alunos e sociedade em geral (TEIXEIRA, 2007). Assim a responsabilidade e exigências projetadas sobre os professores só aumentam (ESTEVE, 1999).

Silva, Carlotto (2003); e Jbeili (2008), acrescentam ainda, que as atividades dos professores geralmente são desenvolvidas em condições potencialmente estressantes, tais como, baixos salários, recursos materiais e didáticos escassos, problemas estruturais nas salas de aula, superlotação, conflitos com alunos, excesso de carga horária e falta de segurança no contexto escolar.

Portanto, nessas condições de trabalho, surge a desmotivação e o estresse, que acabam influenciando no desempenho das atividades e até na qualidade de vida (SANTANA, 2010).

Os professores da rede pública estão mais susceptíveis aos problemas de estresse por conta, entre outras coisas, da exaustão e insatisfação profissional em que geralmente se encontram (LOPES, PONTE, 2009).

Como reflexo desta insatisfação no trabalho, problemas da ordem física e emocional surgem, como é o caso da Síndrome de Burnout. Segundo Jbeili (2008) esta síndrome se "caracteriza pelo estresse crônico vivenciado por profissionais que lidam de forma intensa e constante com as dificuldades e problemas alheios, nas diversas situações".

A Síndrome de Burnout é um problema de saúde relacionado ao trabalho, reconhecida pelo CID (International Statistical Classification of Diseases and Relatend Health Problems/ICD), está cada vez mais presente na vida do trabalhador, sendo considerada como epidemia no meio educacional e um problema de saúde pública (BATISTA, 2010). 
Os estudos sobre esse tema, é uma importante ferramenta de diagnóstico da situação dos professores em todo o país, e muito relevante na avaliação da qualidade do ensino, já que estes profissionais são peça fundamental nesse processo.

Diante desse contexto, esta pesquisa objetivou, avaliar a ocorrência da síndrome de Burnout nos Professores do Ensino Fundamental, do Colégio da Polícia Militar no Vale do São Francisco e suas causas. Este colégio faz parte da Rede Pública de Ensino. Para alcançar este objetivo, o trabalho se propôs a: caracterizar os conceitos que envolvem a Síndrome de Burnout; identificar as condições de trabalho a que estão submetidos os professores envolvidos na pesquisa e detectar o nível de satisfação dos professores respondentes em relação à Qualidade de Vida no Trabalho.

\section{Discussão Teórica}

O trabalho assume dimensões relevantes na vida das pessoas, pois é através dele que elas têm sua subsistência garantida. Porém o trabalho não garante apenas a satisfação das necessidades materiais do homem, mas também as necessidades emocionais, sociais, de autorrealização, autoestima, entre outras.

Segundo Codo et al. (1993, p. 56) "o homem produz sua própria existência, na medida em que trabalha, arquitetando a estrutura social com suas próprias mãos, a mesma estrutura que lhe servirá de habitat; o homem é o meio ambiente do homem".

A palavra trabalho tem diversos significados, podendo ser compreendido como uma forma elementar da ação do homem. É uma atividade determinada e transformadora, muitas vezes penosa, porém necessária (ALBORNOZ, 2008).

A acirrada concorrência imposta pelo mercado capitalista tem exigido dos trabalhadores enorme dedicação e esforço, sendo fato notório que a esmagadora maioria das pessoas passa a maior parte de suas vidas dentro das organizações, em virtude das longas jornadas de trabalho e dos períodos de hora extra. Assim sendo, um trabalho inadequado pode influenciar a qualidade de vida dos profissionais e de sua produtividade (SANTANA, 2010).

Os trabalhadores estão expostos a condições de trabalho que podem ocasionar tensões emocionais, irritações, angustias, síndrome Burnout, doenças cardiovasculares, entre outras. A saúde do funcionário é afetada física ou psicologicamente e por consequência a qualidade do serviço é reduzida, assim como a lucratividade (MORENO-JIMÉNZ, 2000; SCHAUFELI, 1999).

Entre as profissões com elevado risco de tensão e estresse, a docência tem grande destaque, por atuar diretamente com pessoas, pela exigência intrínseca ao papel de professor e pela doação da sua atenção ao cuidado do outro (CARLOTTO, 2011; MANASSERO et al. 2005; SANTOS, LIMA FILHO, 2005), além de outros fatores. Este estresse pode se transformar em problemas físicos e 
psicológicos. Atualmente a síndrome de Burnout é uma das consequências do estresse ocupacional crônico que mais vem acometendo o professor (SILVA et al., 2008).

O Burnout ocorre com mais frequência em profissões em que suas atribuições exigem um constante contato com outras pessoas. Como exemplo as atividades policiais, enfermeiros, professores, terapeutas ocupacionais, psicoterapeutas e outros (FIGUEROA, VELIZ-CAQUIAS, 1992).

Burnout é uma palavra de origem inglesa e significa, Burn "queimar" e Out "para fora", ou seja, consumir-se de dentro para fora. Este termo, Síndrome de Burnout, vem sendo bastante utilizado para designar o estresse relacionado exclusivamente ao ambiente de trabalho.

Segundo Otero et al., (2008) e Silva (2006) nos últimos anos o professor tem sido uma importante vitima desta síndrome, devido entre outros fatores ao desinteresse, à agressividade e à indisciplina dos alunos. As condições de trabalho, como estrutura da escola precária, recursos materiais escassos, bem como a desvalorização salarial, também são grandes propulsores do problema (LEITE, SOUZA, 2007; MANASSERO et al., 2005).

Essa realidade somada a jornada de trabalho em excesso, aos poucos vai se convertendo em desmotivação e esgotamento emocional, podendo ocasionar doenças psicossomáticas e física (MALAGRIS, 2004).

Bomtempo (1999) atribui como causa da síndrome do Burnout, além das já citadas, as questões pessoais, como idealismo inicial e sobrecarga autoimposta.

Lopes e Silva (2009) em estudo comparativo com professores da rede pública e particular, identificou que "professores da escola pública estadual possuem maior exaustão e menor realização profissional que os participantes da rede particular".

Segundo Reinhold (2002) a síndrome de Burnout nos professores pode se desenvolver aos poucos e por fases. O professor inicia sua profissão com muito entusiasmo e energia, cheio de idealismo, mas ao perceber que suas expectativas e ideais estão distantes da realidade, instala-se o sentimento de frustração. Com a intensificação do trabalho em busca de realização, vem o cansaço e a desilusão, acabando o professor por duvidar de sua competência, chegando ao estágio de estagnação e frustração, ou quase-Burnout. É neste momento que surgem sintomas como irritabilidade, fuga dos contatos, atrasos e faltas. A seguir, vem a apatia e Burnout total, situação em que o professor se encontra em desespero, autoestima corroída e até depressão, surge aí o desejo de abandonar o trabalho. A síndrome de Burnout está completa com o fenômeno fênix, que é o renascer das cinzas, mas nem sempre ocorre. Esta última etapa da síndrome, é o momento em que a intervenção externa é essencial para a recuperação e retomada da vida profissional e pessoal, porém muitas vezes o trabalhador mudou de profissão ao longo das fases da síndrome ou simplesmente desistiu da docência.

Segundo Maslach e Jackson (1981), a síndrome de Burnout passa por três dimensões, a exaustão emocional, a despersonalização e a baixa realização profissional. A primeira é o esgotamento da energia devido ao contato diário com os problemas dos alunos; na segunda o professor 
desafeiçoado, passa a ter atitudes pouco positivas com os alunos; no último estágio, ocorre uma percepção da desvalorização e um não reconhecimento do seu trabalho, o que o leva a realizar suas atividades profissionais e pessoais sem habilidade e com muita insatisfação (CODO, VASQUESMENEZES, 1999).

A preocupação dos pesquisadores quanto a questão da síndrome de Burnout em professores, tem rendido muitos estudos no Brasil e no mundo (TOULABI, RAOUFI, ALLAHPOURASHRAF, 2013; CARLOTTO, 2011; BATISTA, CARLOTTO, COUTINHO, AUGUSTO, 2010; CARLOTTO, CÂMARA, 2008; MASLACH, LEITER, 2008; LINDBLOM et al., 2006; LEVY, 2006; MALLAR, CAPITÃO, 2004; MASLACH, SCHAUFELI, 1993).

Em um estudo realizado no Brasil pela UnB, com professores e funcionários da educação da rede pública estadual de todo o país, $48 \%$ dos entrevistados apresentavam algum sintoma da síndrome. A situação nos Estados Unidos é ainda mais grave, de tal forma que as companhias de seguro americanas consideram a profissão de professor como de alto risco (BOCK, SARRIERA, 2006).

Os sintomas da síndrome podem ser de natureza psicossocial e/ou psicossomática. Lipp (2003) afirma que normalmente quem observa os sinais de alarme de Burnout, são os colegas, os amigos e a família.

O reconhecimento da síndrome de Burnout como um problema sério na saúde do professor e na qualidade da educação é o primeiro passo para se lidar com a situação. A escola tem um papel fundamental neste processo, podendo identificar e intervir na rotina do professor para evitar o surgimento da Burnout (TEIXEIRA, 2007), a prática regular de exercícios físicos também pode ajudar na prevenção e intervenção, por exemplo as técnicas de relaxamento melhoram os níveis de pressão arterial, frequência cardíaca, reduz a tensão muscular (MORENO-JIMENEZ, et al., 2002).

\section{Metodologia}

A classificação da pesquisa foi baseada na taxonomia de Vergara (2009) que faz a distinção de dois aspectos: quanto aos fins e aos meios.

Quanto aos fins a pesquisa apresentou natureza exploratória e descritiva. Exploratória pelo fato de não ter ocorrido nenhum estudo no Colégio da Polícia Militar (CPM) que explanasse sobre a existência da Síndrome de Burnout nos professores do Ensino Fundamental. A pesquisa foi descritiva, porque teve o objetivo de descrever as percepções e opiniões do quadro de professores do CPM.

Quanto aos meios a pesquisa foi bibliográfica e de campo. Inicialmente o levantamento de dados se baseou em uma pesquisa bibliográfica, com a necessidade de adquirir um conhecimento mais amplo sobre a Síndrome de Burnout e em busca de considerações já existentes. A pesquisa 
bibliográfica foi realizada através de diversas fontes: livros, revistas, monografias, artigo e pesquisas. De campo, pois, os dados foram coletados no CPM com aplicação de questionários.

De acordo com Vergara (2009, p.43) "pesquisa bibliográfica é o estudo sistematizado desenvolvido com base em material publicado em livros, revistas, jornais, redes eletrônicas, isto é, material acessível ao público em geral”. Segundo Gil (2002), as pesquisas bibliográficas são denominadas de fontes de "papel".

Ainda de acordo com Vergara (2009) pesquisa de campo é realizada no local onde ocorre ou ocorreu um fenômeno que contenham elementos para explicá-lo, pode ser feito através de aplicação de questionários por exemplo.

A população-alvo da pesquisa foram os 28 professores do Ensino Fundamental que compõem o quadro de funcionários do CPM no Vale do São Francisco.

Instrumento de coleta de dados

Como instrumento básico de coleta e levantamento de dados foi aplicado um questionário que atenda a finalidade desta pesquisa, o qual foi respondido individualmente pelos professores.

Para Marconi e Lakatos (2010, p. 184) "questionário é um instrumento de coleta de dados, constituído por uma série ordenada de perguntas, que devem ser respondidas por escrito e sem a presença do entrevistador". Os questionários foram entregues pessoalmente e foram devolvidos respondidos aos pesquisadores.

Esse instrumento apresenta uma série de vantagens segundo Marconi e Lakatos (2010), dentre as quais se destacam as seguintes: economia de tempo; atinge maior número de pessoas simultaneamente; consegue respostas mais rápidas; há mais segurança, em virtude de as respostas não serem identificadas.

Para avaliar a síndrome de Burnout, foi utilizado o questionário MBI-ED-Maslash Burnout Inventory - Educators Survey (MASLACH; JACKON; LEITER, 1996), versão para professores, com adaptação para uso no Brasil.

Com o intuito de preservar a identidade dos professores pesquisados e a ética da pesquisa, optamos pela utilização de números para identificação dos professores respondentes.

Para a análise dos resultados, foi feito um levantamento das respostas dadas a cada questão respondida pelos professores e posteriormente um comparativo com as diversas citações na literatura levantada. 


\section{Análise de Dados}

O Colégio analisado possui atualmente 28 professores do Ensino Fundamental e todos eles participaram da pesquisa. Primeiramente são apresentados os dados relacionados aos aspectos profissionais e pessoais dos professores, visando entender o perfil dos participantes da pesquisa.

- Quanto a faixa etária dos professores: 50\% têm entre 31 e 40 anos; $36 \%$ têm de 41 a 50 anos; $7 \%$ têm entre 18 e 30 anos; e 7\% está acima dos 50 anos.

- $\quad$ Dos 28 professores, $32 \%$ são do sexo masculino, e 68\% são do sexo feminino.

- Em relação ao grau de escolaridade, a maior parte dos professores possui especialização (64\%), seguido de nível superior completo (25\%), mestrado, superior incompleto e médio completo (4\% cada). Foi verificado que nenhum professor possui a titulação de doutorado.

- Por fim, quanto ao tempo de serviço no CPM, $54 \%$ dos professores trabalham na instituição de 05 a 10 anos, 39\% de 01 a 05 anos e $7 \%$ menos de 01 ano.

No que se refere ao levantamento de dados quanto a existência da Síndrome de Burnout nos professores do CPM, a avaliação levou em consideração, algumas variáveis, tais como, a síndrome sem distinção de sexo e idade, o sexo e a idade dos docentes entrevistados.

Analisando a tabela 1, nota-se um sinal de alerta para a escola CPM quanto ao problema da síndrome de Burnout, já que, do quadro de professores, $43 \%$ apresentaram possibilidade de desenvolver a síndrome, necessitando, portanto, trabalhar as recomendações de prevenção. E há ainda um número maior de professores (46\%) em que já se encontram na fase inicial da Burnout, ou seja, este grupo precisa de ajuda profissional na superação dos sintomas, garantindo, a qualidade no desempenho profissional e pessoal. Os outros $11 \%$ dos professores encontram-se em fases mais avançadas da síndrome, sendo que em $4 \%$ deles a Burnout começa a se instalar, precisando de ajuda profissional para evitar o agravamento dos sintomas e os outros 7\% podem estar em uma fase considerável da Burnout, o tratamento para os profissionais que se encontram nesta fase deve iniciar o mais rápido possível. Estes dados demonstram que os professores da escola CPM estão desenvolvendo suas atividades em condições que possibilitam o surgimento de estresse e aos poucos da síndrome de Burnout. O que é perfeitamente justificável, tendo em vista que mais da metade (54\%) dos profissionais avaliados, trabalham na Escola Militar entre 05 e 10 anos, lidando intensivamente e constantemente com as dificuldades e problemas alheios o que os coloca sob condições de estresse crônico, que é considerado por Jbeili (2008) como a caracterização da síndrome de Burnout.

De acordo com Silva, Carlotto (2003) e Jbeili (2008), os baixos salários, recursos materiais e didáticos escassos, problemas estruturais nas salas de aula, superlotação, conflitos com alunos, excesso de carga horária e falta de segurança no contexto escolar, são alguns dos fatores estressantes mais significativos. 
Tabela 1 - Resultados sem distinção de sexo e idade

\begin{tabular}{l|c}
\multicolumn{1}{c|}{ Pontuação } & $\begin{array}{c}\text { Percentuais de } \\
\text { Professores nesta } \\
\text { situação }\end{array}$ \\
\hline De 0 a 20 pontos: Nenhum indício da Burnout. & $0 \%$ \\
\hline De 21 a 40 pontos: Possibilidade de desenvolver Burnout. & $43 \%$ \\
\hline De 41 a 60 pontos: Fase inicial da Burnout. & $46 \%$ \\
\hline De 61 a 80 pontos: A Burnout começa a se instalar. & $4 \%$ \\
\hline $\begin{array}{l}\text { De 81 a 100 pontos: Você pode estar em uma fase considerável da } \\
\text { Burnout. }\end{array}$ & $7 \%$ \\
\hline
\end{tabular}

Quanto a avaliação da síndrome de Burnout, a tabela 2 distingue os docentes pela variável sexo. Avaliando os resultados dos professores do sexo masculino, nota-se que $67 \%$ dos professores estão na fase inicial da Burnout, fase em que a qualidade de vida, bem como o bom desempenho profissional podem ser comprometidos pelos sintomas da síndrome, e para evitar tais implicações estes professores precisam de ajuda de profissionais competentes. Os outros $33 \%$ estão divididos igualmente entre a possibilidade de desenvolver Burnout, a fase em que a síndrome começa a se instalar e a fase em que o professor já pode estar em uma fase considerável da Burnout.

Analisando apenas os resultados dos professores do sexo feminino, observa-se que 58\% dos professores apresentaram possibilidade de desenvolver Burnout, 37\% estão na fase inicial da Burnout, enquanto que os outros 5\% podem estar em uma fase considerável da Burnout. Percebemos que não houve nenhum professor que estivesse nas demais faixas. Dessa forma, é possível observar que a maioria dos professores do sexo feminino tem possibilidade de desenvolver Burnout, sendo possível evitar a instalação da síndrome, com medidas de prevenção.

Assim quanto à variável sexo, os homens apresentam mais chance de desenvolver a síndrome do que as mulheres, nota se ainda que, de acordo com o teste aplicado, o número de professores do sexo masculino que já podem estar em uma fase considerável da síndrome é o dobro do número dos professores do sexo feminino.

Entretanto, o estudo revelou um número maior de mulheres em relação aos homens que já estão na fase inicial da síndrome. Este último dado indica que enquanto os homens já estão em uma fase avançada da Burnout, as mulheres estão iniciando o processo da síndrome.

De acordo Farber (1991), os professores do sexo masculino são mais vulneráveis que os do sexo feminino. Possivelmente isso se deva ao fato das mulheres desempenharem e acumularem na grande maioria das vezes, mais papéis na sociedade (trabalho, família, entre outros) que os homens, ela divide a sua atenção com todas as tarefas e adapta-se melhor as pressões. Etzion (1987) relaciona as diferenças encontradas nos níveis do Burnout às questões tradicionais do processo de socialização e organização social, as quais se colocam diferenciadamente para homens e mulheres. 
Tabela 2 - Resultados distinguindo por Sexo

\begin{tabular}{l|c|c}
\hline \multicolumn{1}{c|}{ Pontuação } & \multicolumn{2}{c}{$\begin{array}{c}\text { Percentuais de } \\
\text { Professores nesta } \\
\text { situação }\end{array}$} \\
\cline { 2 - 3 } & Masculino & Feminino \\
\hline De 0 a 20 pontos: Nenhum indício da Burnout. & $0 \%$ & $0 \%$ \\
\hline De 21 a 40 pontos: Possibilidade de desenvolver Burnout. & $11 \%$ & $58 \%$ \\
\hline De 41 a 60 pontos: Fase inicial da Burnout. & $67 \%$ & $37 \%$ \\
\hline De 61 a 80 pontos: A Burnout começa a se instalar. & $11 \%$ & $0 \%$ \\
\hline $\begin{array}{l}\text { De 81 a 100 pontos: Você pode estar em uma fase considerável } \\
\text { da Burnout. }\end{array}$ & $11 \%$ & $5 \%$ \\
\hline
\end{tabular}

Quanto a variável idade, a tabela 3, mostra a sensibilidade dos professores a Burnout de acordo com a idade. As faixas etárias avaliadas foram, 18 a 30 anos; 31 a 40 anos; 41 a 50 anos e acima de 50 anos.

O resultado do grupo de professores entre 18 e 30 anos apresenta o mesmo comportamento, 100\% mostram possibilidade de desenvolver Burnout. Não houve nenhum professor que estivesse nas outras faixas. Este resultado pode não ser tão expressivo visto que esta faixa etária é composta apenas por dois professores.

Nos professores com idade entre 31 e 40 anos verificou-se que $57 \%$ dos professores desta faixa etária estão na fase inicial da Burnout, $36 \%$ estão com possibilidade de desenvolver Burnout e em $7 \%$ a Burnout começa a se instalar.

Avaliando os resultados dos professores com idade entre 41 e 50 anos, é possível perceber que $50 \%$ dos professores estão na fase inicial da Burnout, $40 \%$ estão com possibilidade de desenvolver Burnout e 10\% podem estar em uma fase considerável da Burnout. Não houve nenhum professor que estivesse nas demais fases da síndrome.

Analisando os resultados dos professores com idade acima de 50 anos. Desta faixa etária 50\% estão na fase inicial da Burnout e os outros 50\% podem estar em uma fase considerável da Burnout. Não houve nenhum professor que estivesse nas demais faixas.

Diante dos dados coletados, identificamos que todas as faixas etárias apresentaram possibilidades de desenvolverem ou já estão em alguma das fases da síndrome de Burnout. Entretanto nota-se um agravamento da síndrome de Burnout com a idade do professor, ou seja, a medida em que aumenta a faixa etária, tem-se uma percentagem maior de professores nas fases mais críticas da síndrome. Enquanto todos professores da faixa etária 18 a 30 anos, apenas mostram-se susceptíveis a desenvolver Burnout, os professores que estão na faixa etária 41 a 50 anos e acima de 50 anos, ou estão na fase inicial, na fase de instalação ou em uma fase bem mais avançada da Burnout. 
Estes resultados indicam que quanto maior a idade do professor mais crítica está a sua situação quanto a síndrome de Burnout. O que pode estar relacionado ao cansaço deste profissional e ao sucateamento da profissão.

Diferente do que indica os resultados desta pesquisa, Carlotto (2002) afirma que professores com menos de 40 anos apresentam maior risco de adquirir a Burnout, devido às expectativas irrealistas em relação à profissão.

Resultado diferentes também foram encontrados por Valerio, Amorim, Moser (2009), em estudo realizado com professores de educação física, encontram maior número de professores com Burnout na faixa etária de 31 a 40 anos.

Tabela 3 - Resultados distinguindo por Faixa Etária

\begin{tabular}{l|c|c|c|c}
\hline \multirow{2}{*}{ Pontuação } & \multicolumn{3}{|c}{ Percentuais de Professores nesta situação } \\
\cline { 2 - 5 } & De 18 a 30 & De 31 a 40 & De 41 a 50 & Acima de 50 \\
\hline $\begin{array}{l}\text { De 0 a 20 pontos: Nenhum indício da } \\
\text { Burnout. }\end{array}$ & $0 \%$ & $0 \%$ & $0 \%$ & $0 \%$ \\
\hline $\begin{array}{l}\text { De 21 a 40 pontos: Possibilidade de } \\
\text { desenvolver Burnout. }\end{array}$ & $100 \%$ & $36 \%$ & $50 \%$ & $0 \%$ \\
\hline $\begin{array}{l}\text { De 41 a 60 pontos: Fase inicial da } \\
\text { Burnout. }\end{array}$ & $0 \%$ & $57 \%$ & $40 \%$ & $50 \%$ \\
\hline $\begin{array}{l}\text { De 61 a 80 pontos: A Burnout começa } \\
\text { a se instalar. }\end{array}$ & $0 \%$ & $7 \%$ & $0 \%$ & $0 \%$ \\
\hline $\begin{array}{l}\text { De 81 a 100 pontos: Você pode estar } \\
\text { em uma fase considerável da Burnout. }\end{array}$ & $0 \%$ & $0 \%$ & $10 \%$ & $50 \%$ \\
\hline
\end{tabular}

\section{Conclusões}

A grande responsabilidade social que está inserida no processo de educar é o que faz da docência uma profissão relevante a todos os seguimentos da vida e que idealmente deveria ter as melhores condições de trabalho. Como na prática não ocorre assim, as pesquisas voltadas para avaliar estas condições, o nível de estresse e o reflexo disto na sociedade é uma ferramenta importante para apontar os erros no sistema educacional e indicar as soluções para a sua melhoria.

O desenvolvimento desta pesquisa permitiu avaliar a saúde dos professores do ensino fundamental da escola CPM, identificando as categorias mais susceptíveis ao estresse e a síndrome de Burnout.

Assim, inferi-se que a escola estudada apresenta um elevado percentual de professores em fase inicial da síndrome de Burnout ou susceptíveis de desenvolverem a síndrome. Isso pode ter relação 
com a desvalorização profissional e pessoal, porém faz-se necessário estudos mais detalhados que avaliem e correlacione estes fatores para um diagnóstico mais exato.

Dos professores avaliados, os do sexo masculino apresentaram maior predisposição a desenvolverem a Burnout, que os do sexo feminino. Apesar de estarem nas mesmas condições de trabalho, as mulheres conseguem lidar melhor com as situações desfavoráveis.

Quanto a idade, quão maior a idade do professor, mais crítica a fase da Burnout em que se encontra. Acima de 40 anos, $40 \%$ dos avaliados estão iniciando a síndrome, quando a idade ultrapassa os 50 anos, $50 \%$ deles estão na fase mais crítica e precisam de tratamento imediato.

Considerando estes altos percentuais de professores com Burnout ou susceptível a ela, fica claro que estes docentes necessitam de ajuda profissional para controlar e superar os sintomas, evitando que o ensino seja comprometido e não permitindo redução na qualidade de vida do professor.

Diante dos resultados obtidos, percebe-se a necessidade de estudos complementares que abordem esse tema e seus fatores de influência.

Sugere-se ainda a implantação pelo sistema educacional de programas que busquem evitar o desenvolvimento da Burnout nos professores e/ou que ajudem a controlar e eliminar os sintomas. Projetos modelos que façam estudos das condições de trabalho, inserindo mudança na rotina desses profissionais, como diminuição na carga horária, incentivo para capacitações, apoio da comunidade, e até mesmo inclusão de exercícios para redução do nível de tensão e estresse.

\section{Referências}

ALBORNOZ, Suzana. O que é trabalho. Coleção Primeiros Passos. São Paulo: Brasiliense, 2008.

BATISTA, J. B. V. Síndrome de Burnout em professores do ensino fundamental: um problema de saúde pública não percebido. Tese (Doutorado em Saúde Pública) - Centro de pesquisas Aggeu Magalhães, Fundação Oswaldo Cruz, Recife - PE. 2010.

BATISTA, J. B. V., CARLOTTO, M. S., COUTINHO, A. S., AUGUSTO, L. G. da S. Prevalência da Síndrome de Burnout e fatores sociodemográficos e laborais em professores de escolas municipais da cidade de João Pessoa, PB. Revista Brasileira de Epidemiologia, 13(3), 502-512, 2010.

BOCK, V. R., SARRIERA, J. C. O grupo operativo intervindo na Síndrome de Burnout. Psicologia escolar e educacional, 10(1), 31-39, 2006.

BONTEMPO, X. F. Nível de síndrome de adotamiento em médicos, enfermeras y paramédicos. Revista Mex de Puericultura y Pediatria. v. 6, n.2, p. 252-260, 1999.

CARLOTTO, M. S. Síndrome de Burnout em Professores: Prevalência e Fatores Associados. Psic.: Teor. e Pesq., Brasília, Out-Dez 2011, Vol. 27 n. 4, pp. 403-410. 
CARLOTTO, M. S., CÂMARA, S. G. Análise da produção científica sobre a Síndrome de Burnout no Brasil. Psico-PUCRS, 39(2), 152-158, 2008.

CARLOTTO, M. S. C. A síndrome de Burnout e o trabalho docente. Psicologia em Estudo, Maringá, v. 7, n. 1, p. 21-29, jan./jun. 2002.

CODO, W., SAMPAIO, J. J. C., HITOMI, A. H. Indivíduo, Trabalho e Sofrimento: uma abordagem interdisciplinar. Petrópolis: Vozes, 1993.

CODO, W.; VASQUES-MENEZES,I. V. O que é Burnout? In: Codo (coord.) 1999. Educação carinho e trabalho. Burnout, a síndrome da desistência do educador, que pode levar à falência da educação. Petrópolis: Vozes, CNTE e UNB. 1999. p.237-254.

ESTEVE, J..M. O mal-estar docente: a sala de aula e a saúde dos professores. São Paulo: EDUSC, 1999.

ETZIN, D. (1987). Burning out in management: a comparison of woman and men in matched organizational positions. Israel Social Science Research, 5, 1\&2, 147-163.

FARBER, B. A. (1999). Inconsequentiality_- The key to understanding teacher Burnout. Em Vanderbergue, R. \& Huberman, M. A. (Eds.), Understanding and preventing teacher Burnout: a source book of international practice and research (pp.159).

FIGUEROA, J. F.; VELIZ-CAQUIAS, R. E. El Síndrome de quemazón y el ambiente de trabajo em uma muestra de enfermeras psiquiátricas. Rev. Ciencias de la Conducta. n. 3, p. 101-121, 1992.

GIL, A. C. Como elaborar projetos de pesquisa. 4. ed. São Paulo: Atlas, 2002.

JBEILI, C. Sindrome de Burnout: Identificação, tratamento e prevenção. Cartilha informativa de prevenção à Síndrome de Burnout em professores, Brasília-DF, 2008.

LEITE, M DE P. SOUZA, A. N. de. Condições do trabalho e suas repercussões na saúde dos professores da educação básica no Brasil-Estado da Arte. São Paulo: Fundacentro/Unicamp, 2007.

LEVY, G. C. T. de M. Avaliar a síndrome de Burnout em professores da rede pública de ensino. Dissertação de Mestrado, Universidade do Estado do Rio de Janeiro, Rio de Janeiro, 2006.

LINDBLOM, K. M., LINTON, S. J., FEDELI, C., BRYNGELSSON, I-L. Burnout in the Working Population: Relations to Psychosocial Work Factors. International Journal of Behavioral Medicine, 13(1), $51-59,2006$.

LIPP, M. O stress do professor._Campinas: Papirus, 2003.

LOPES, A. P., PONTES, E. A. S. Síndrome de Burnout: um estudo comparativo entre professores das redes pública estadual e particular. Revista Semestral da Associação Brasileira de Psicologia Escolar e Educacional (ABRAPEE). Vol.13, N 2, 275-281, Julho/Dezembro de 2009.

MALAGRIS, L. E. N. Burnout: o profissional em chamas. In: NUNES SOBRINHO, F. de P.; NASSALLA, I. (Orgs.). Pedagogia Institucional: fatores humanos nas organizações. Rio de Janeiro: ZIT Editores, 2004. p. 196-213. 
MALLAR, S. C., CAPITÃO, C. G. Burnout e hardiness: Um estudo de evidência de validade. PsicoUSF, 9(1), 19-29, 2004.

MANASSERO, M. A., GARCÍA, E., TORRENS, G., RAMIS, C., VÁZQUEZ, A., FERRER, V. A. Burnout en la enseñanza: aspectos atribucionales. Revista de Psicología del Trabajo y de las Organizaciones, 21(1-2), 89-105, 2005.

MARCONI, M. de A.; LAKATOS, E. M. Fundamentos de metodologia científica. 7. ed. São Paulo: Atlas, 2010.

MASLACH, C.; JACKSON, S. The Measurement of Experienced Burnout. Journal of Occupational Behavior, 1981.

MASLACH, C.; JACKON, S. E.; LEITER, M. P. Burnout Inventory Manual. Palo Alto, C. A: Consulting Paychologist Press; 1996.

MASLACH, C., LEITER, M. P. Early predictors of job Burnout and engagement. Journal of Applied Psychology, 93(3), 498-512, 2008.

MASLACH, C., SCHAUFELI, W. B. Historical and conceptual development of Burnout. Em W.B. Schaufeli, C. Maslach \& T. Marek (Eds.), Professional Burnout: recent developments in theory and research (pp.1-16). New York: Taylor \& Francis, 1993.

MORENO-JIMENEZ, B. et al. A avaliação do Burnout em professores. Comparação de instrumentos: CBP-R e MBI-ED. Psicologia em estudo, Maringá, v. 7, n. 1, jan./jun, 2002.

MORENO-JIMÉNEZ, B. Olvido y recuperación de los factores psicosociais en la salud laboral. Archivos de Prevención de Riesgos Laborales, Madrid, v. 3, n. 1, p. 3-4,jan./mar. 2000.

OTERO, L. J. M., SANTIAGO, M. J., GODÁS, A., CASTRO, C., VILLAEDEFRANCOS, E.,

PONTE, D. An integrative approach to Burnout in secondary school teachers: examining the role of student disruptive behavior and disciplinary issues. International Journal of Psychology and Psychological Therapy, 8(2), 259-270, 2008.

REINHOLD, H. H. Burnout. In: LIPP, M. E. N. O stress do professor. Campinas: Papirus, p. 63-80, 2002.

RUBEGA, C. C., PACHECO, D. A formação da mão-de-obra para a indústria química: uma retrospectiva histórica. Ciência \& Educação, v. 6, n. 2, p. 151-166, 2000.

SANTANA, Ana Cristina de Araujo Souza. Qualidade de Vida no Trabalho na Percepção dos Servidores do Departamento de Desenvolvimento e Administração de Recursos Humanos da Universidade Federal do Vale do São Francisco. 2010. 66. Curso de Pós-Graduação lato sensu: Gestão em Organizações Públicas. Universidade do Vale do São Francisco, Petrolina-PE.

SANTOS, F. L. N., LIMA FILHO, D. L. Mudanças no Trabalho e Adoecer Psíquico na Educação. Universia Rede Universitária, 2005.

SANTOS, H. C. dos. Formação de professores: abordagens conceituais e realidade de algumas práticas realizadas no contexto brasileiro. Revista Estação Científica - Juiz de Fora, no 09, janeiro junho / 2013. 
SCHAUFELI, W. Evaluación de riesgos psicosociales y prevención del estrés laboral: algunas experiencias holandesas. Revista de Psicologia del Trabajo y de las Organizaciones, Madrid, v. 15, n. 2, p. 147-171, 1999.

SILVA, G. N., CARLOTTO, M. S. Síndrome de Burnout: Um estudo com professores da rede pública. Psicologia Escolar Educacional, 7(2), 145-153, 2003.

SILVA, J. P. da, DAMÁSIO, B. F., MELO, S. A., AVELAR, T. A. A. de. Estresse e Burnout em professores. Revista Fórum Identidades. Vol. 3 - p. 75-88 -jan-jun, 2008.

SILVA, M. E. P. Burnout: por que sofrem os professores? Estudos e pesquisas em psicologia. Programa de Pós-Graduação em Políticas Públicas e Formação Humana-UERJ, 2006.

TEIXEIRA, G. F. Síndrome de Burnout em profissionais da educação: um estudo com professores da rede municipal do ensino fundamental de Rio Grande/RS. Dissertação (Mestrado em Enfermagem/Saúde - Fundação Universidade Federal), Rio Grande/RS, 2007.

TOULABI, Z., RAOUFI, M., ALLAHPOURASHRAF, Y. The Relationship Between Teachers, Happiness and Quality of Working Life. Procedia - Social and Behavioral Sciences,84, 691- 95, 2013.

VALERIO, F. J., AMORIM, C., MOSER, A. M. A síndrome de Burnout em professores de educação física. Revista de Psicologia da IMED, vol. 1, n. 1, p.127-136. 2009

VERGARA, Sylvia Constant. Projetos e relatórios de pesquisa em administração. 10. ed. São Paulo: Atlas, 2009.

\section{Como citar este artigo (Formato ABNT):}

SILVA, Quenia L da; MENEZES, Thiago Félix de A.; CASSUNDÉ, Fernanda R. de S. A. Esgotamento Psicológico no Trabalho: uma análise sob a ótica da Síndrome de Burnout em Professores do Ensino Fundamental. Id on Line Revista de Psicologia, Fevereiro de 2016, vol.10, n.29. p. 37-50. ISSN 1981-1179.

Recebido: 12/02/2016.

Aceito: 26/02/2016 